Befassten sich schulärztliche Dienste zu Beginn, d.h. nach Einführung des Schulobligatoriums 1874, hauptsächlich mit Haltungsschäden und den richtigen Massen von Schulbänken, ist das heutige Aufgabenspektrum einiges umfassender. Bei u.a. Früherfassung oder individueller Beratung, in den Bereichen Epidemiologie, Impfungen, Erfassung gesundheitlicherTrends und der Mitarbeit in der schulischen Gesundheitsförderung sind Schulärzte/-ärztinnen ein wichtiges Bindeglied zwischen Individualmedizin und Public Health. Sie haben eine wichtige Public-H ealth-Rolle. Der vorliegende Artikel gibt einen Überblick zu kantonalen Heterogenitäten und das Potential künftiger Ausgestaltung schulärztlicher Dienste. Wesentlich ist die gute Zusammenarbeit und Koordination zwischen Schulärzten/ärztinnen und Haus- und Kinderärzten/-ärztinnen im jeweiligen Kanton, wobei in einigen Kantonen Doppelrollen von der Ärzteschaft wahrgenommen werden.

\title{
Organisation der schulärztlichen Untersuchungen in der Schweiz
}

\section{Fabienne Lüthi ${ }^{a}$, Andreas Balthasar ${ }^{b}$, Birgit Laubereau ${ }^{c}$}

${ }^{a}$ BSc in Physiotherapie, MA Health Sciences and Health Policy, Projektleiterin, physioswiss; ${ }^{b}$ Prof. Dr., Seminar für Gesundheitswissenschaft und Gesundheitspolitik, Universität Luzern; ${ }^{\mathrm{C}}$ Dr. med. und MPH, Projektleiterin, Interface Politikstudien Forschung Beratung

\section{Zusammenfassung}

Schulärztliche Dienste können mit ihrem vielseitigen Aufgabenspektrum und der umfassenden Reichweite wichtige Funktionen bei der Förderung der Gesundheit von Kindern und Jugendlichen übernehmen. In der Schweiz haben sich die schulärztlichen Dienste in den Kantonen sehr unterschiedlich entwickelt. Ziel der Studie ist es, die kantonale Heterogenität der schulärztlichen Untersuchungen aufzuzeigen und darauf aufbauend Modelltypen zu bilden, welche als Grundlage für weitergehende Arbeiten dienen können. Weitere wichtige Public-Health-Funktionen (z.B. Unterstützung der Schulleitungen mit Public-Health-Fragen) der schulärztlichen Dienste sind nicht Gegenstand der vorliegenden Analyse.

Für die Charakterisierung der Organisation von schulärztlichen Untersuchungen werden vier Kriterien definiert: Rolle der schulärztlichen Dienste, Umfang der Untersuchungen, Bedeutung von Impfungen sowie die Qualifikationen des involvierten Personals. Basierend auf diesen Kriterien werden vier Organisationsmodelle definiert, die sich betreffend Häufigkeit und Umfang der Untersuchungen sowie involviertes Personal unterscheiden. Zudem wird die Rolle der schulärztlichen Dienste berücksichtigt, d.h. die Tatsache, ob diese Untersuchungen von den Diensten selbst durchgeführt werden oder ob sie an die Grundversorger delegiert werden. Die im Rahmen der Studie durchgeführten Recherchen deuten auf Diskussionsbedarf bezüglich Zielsetzung und Organisation der schulärztlichen Untersuchungen hin. Zudem wären mit Blick auf das Potential und die zukünftige Ausgestaltung schulärztlicher Dienste Analysen zur Wirksamkeit und Effizienz der verschiedenen Organisationsmodelle von grossem Interesse.
In der Schweiz sind Schulärztinnen und Schulärzte respektive die schulärztlichen Dienste seit der zweiten Hälfte des 19. Jahrhunderts ein wichtiger Bestandteil der Förderung, des Erhalts und des Schutzes der Kinder- und Jugendgesundheit [1-3]. An der Schnittstelle von Bildung und Gesundheit haben sie einen breiten Zugang zu allen Kindern und Jugendlichen in der Schweiz und können deren Entwicklung auf gesundheitlicher Ebene unterstützen.

Die Zuständigkeit für die schulärztlichen Dienste liegt im föderalen System der Schweiz bei den Kantonen und den Gemeinden. Bislang existiert kein Überblick über die Organisation und die Leistungen schulärztlicher Dienste in den Kantonen der Schweiz. Ein solcher Überblick ist jedoch wichtig, weil die Bedeutung der schulärztlichen Dienste in den letzten Jahren zunehmend öffentlich diskutiert wird [4-7]. Die vorliegende Arbeit trägt dazu bei, diese Lücke zu füllen.

Ziel der Studie ist es, die kantonale Heterogenität schulärztlicher Untersuchungen aufzuzeigen. Darauf aufbauend werden Organisationsmodelltypen gebildet, welche als Grundlage für weitergehende Arbeiten dienen können. Die Arbeit fokussiert auf den Vergleich schulärztlicher Untersuchungen einschliesslich allfälliger Impfungen. Die weiteren wichtigen Funktionen der schulärztlichen Dienste sind nicht Gegenstand der Analyse (z.B. die Beratung von Eltern und Lehrpersonen, die Koordination von Informationsflüssen, die 
Unterstützung der Schulleitung in Public-Health-Fragen und das Monitoring von gesundheitlichen Trends sowie die Identifizierung von allfälligem Handlungsbedarf). Auch wird der Umstand nicht berücksichtigt, dass Organisation und Umfang schulärztlicher Untersuchungen einzelner Gemeinden von der kantonalen Praxis abweichen können.

\section{Methodik}

Zentrale Datengrundlage bildete eine systematische Internetrecherche zu den schulärztlichen Diensten in allen Kantonen (Stand Dezember 2016). Die erhobenen Parameter sind nachfolgend in der Tabelle 1 aufgelistet. Davon ausgehend wurden gemeinsam mit Expertinnen und Experten vier Dimensionen zur Charakterisierung der Organisation von schulärztlichen Untersuchungen identifiziert. Es sind dies (1) die Rolle der schulärztlichen Dienste, (2) der Untersuchungsumfang, (3) die Bedeutung von Impfungen und (4) die Qualifikationen des involvierten Personals [8]. Im Folgenden wurde die Recherche punktuell mittels telefonischer Rückfragen bei den zuständigen Stellen vertieft. Dabei zeigte sich, dass die Praxis von den verfügbaren Informationen im Internet abweichen kann. Abschliessend wurden vier Modelltypen zur Beschreibung der Organisation von schulärztlichen Untersuchungen gebildet. Um mögliche Abweichungen der verfügbaren Informationen im Zeitraum zwischen Dezember 2016 und der Publikation zu identifizieren, empfiehlt es sich, die Kantone direkt anzugehen. Die Internetseite der Schweizerischen Vereinigung der Fachpersonen im schulärztlichen Dienst (www.scolarmed.ch) bietet hierfür einen guten Zugang.

Tabelle 1: Auflistung der erhobenen Parameter.

Gesetzliche Grundlage (Gesundheits- und/oder Schulgesetz)

Verordnungen, Weisungen

Zuständige Instanz auf kantonaler Ebene

Auftraggeber/administrative Aufsicht

Zuständige Instanz für die Ernennung/Wahl des schulärztlichen Dienstes

Fachliche Aufsicht

Impfungen

Anzahl der Untersuchungen

Inhalt der Untersuchung im Kindergarten

Inhalt der Untersuchung auf Primarstufe

Inhalt der Untersuchung auf Sekundarstufe

Ausführendes/involviertes Personal

Qualifikation des Personals

Freie Arztwahl ja/nein

Angaben zur Finanzierung

Weitere Besonderheiten
Eine umfassende Darstellung der Methodik findet sich in der zugrundeliegenden Masterarbeit an der Universität Luzern [9].

\section{Resultate}

\section{Vier Dimensionen der Organisation von schulärztlichen Untersuchungen}

\section{Rolle der schulärztlichen Dienste}

In den meisten Kantonen werden Schulärzte/-innen beauftragt, die Kinder in ihren Praxen oder in den Schulen zu untersuchen. Acht Kantone haben die schulärztlichen Untersuchungen in den privaten Sektor an die Praxen von Grundversorgern (Kinder- oder Hausarzt/-ärztin) ausgelagert. Einige Kantone üben dabei eine Aufsichtsfunktion aus (Graubünden, Neuenburg, Nidwalden, Solothurn). Im Kanton Solothurn z.B. müssen zwei Kontrollen bei Haus- bzw. Kinderärztin/Kinderarzt durchgeführt werden. Die Schulärztin/der Schularzt überprüft die Bescheinigungen über die Durchführung der Kontrollen. Vorteil dieses Systems ist, dass in diesen Kantonen Überschneidungen mit den pädiatrischen Vorsorgeuntersuchungen verringert werden, da die Kinder und Jugendlichen von den individuell ausgewählten Grundversorger/ -innen untersucht werden. Nachteil ist die Gefahr, dass die Gesundheit von Kindern und Jugendlichen nicht regelmässig überprüft wird, wenn sie keinen Grundversorger aufsuchen. Dieses Problem stellt sich insbesondere in jenen Kantonen, in denen die Durchführung der Untersuchung nicht überprüft wird (Genf, Obwalden, Tessin, Waadt). In den analysierten Dokumenten fanden sich nur selten konkrete Beschreibungen der Ziele und der Aufgaben der schulärztlichen Dienste.

\section{Untersuchungsumfang}

Die Häufigkeit der Untersuchungen während der obligatorischen Schulzeit (1. Jahr Kindergarten bis zum 9. Schuljahr) variiert gemäss Dokumentation im Internet deutlich zwischen den Kantonen. In elf Kantonen sind drei oder vier Untersuchungen durch den schulärztlichen Dienst vorgesehen (Kindergarten/1. Primar 3.-5. Primar; 7.-9. Sekundar), in sechs Kantonen zwei und in Schaffhausen eine Untersuchung (Kindergarten). In den acht Kantonen, welche die Untersuchungen in den privaten Sektor ausgelagert haben, werden die Eltern in der Regel eingeladen, mit dem Kind ihren Grundversorger aufzusuchen.

In fast allen Kantonen wird eine Art Basisuntersuchung durchgeführt. Hierzu zählen das Messen von Gewicht und Grösse sowie das Testen von Hör- und 
Sehvermögen. In einem Kanton wird einzig diese Basisuntersuchung durchgeführt (Wallis). In fünf Kantonen wird diese Basisuntersuchung durch eine Untersuchung des Rückens bezüglich Wirbelsäulenfehlentwicklungen (Skoliosen) ergänzt. In 12 Kantonen wird zudem ein Beratungsgespräch zu Gesundheitsthemen in der Sekundarstufe verlangt. In 14 Kantonen werden zusätzliche Untersuchungen angeboten, wie die Auskultation von Herz und Lunge, die Messung von Puls und Blutdruck oder die Beurteilung des Entwicklungszustandes, der Sprache und der Grob- und Feinmotorik.

\section{Bedeutung von Impfungen}

In allen Kantonen ist die Kontrolle der Impfdokumente oder zumindest eine Aufforderung dazu, dies durch den Grundversorger vornehmen zu lassen, vorgesehen. Bei Bedarf werden die Eltern über Impflücken informiert. In 16 Kantonen wird zur Schliessung der Impflücken auch die Durchführung von empfohlenen Basisimpfungen durch den schulärztlichen Dienst im Rahmen der schulärztlichen Untersuchungen angeboten. In zehn Kantonen besteht diese Möglichkeit nicht. In einigen Kantonen werden auch spezifische Impfungen (HPV und/oder Hepatitis B) durch die schulärztlichen Dienste im Rahmen von kantonalen Impfprogrammen angeboten (z.B. Luzern, Baselland, Genf). Wie in anderen Studien gezeigt wurde, bestehen grosse kantonale Unterschiede bei der Organisation und Umsetzung der kantonalen HPV-Impfprogramme. Es wurde festgestellt, dass in den Kantonen, in denen die schulärztlichen Dienste diese Impfungen anbieten, die Durchimpfungswerte nicht nur für HPV, sondern auch für andere Impfungen tendenziell höher sind [10].

\section{Qualifikation des involvierten Personals}

In 17 Kantonen werden die schulärztlichen Untersuchungen durch von der Gemeinde beauftragte Schulärztinnen oder Schulärzte erbracht, welche in der Regel von einer Praxisassistenz unterstützt werden. Die meisten Schulärzte/-innen sind nebenamtlich tätig, in grösseren Städten gibt es zum Teil auch hauptamtliche Schulärzte/-innen. In den schulärztlichen Diensten sind häufig auch andere Fachpersonen und administratives Personal tätig. So verabreichen beispielsweise in den Kantonen Aargau und Jura Pflegefachpersonen in Delegation die Impfungen, und im Kanton Obwalden bearbeiten administrative Mitarbeiter/-innen die Vergütung der Grundversorger für die ausgelagerten Untersuchungen. In den Westschweizer Kantonen (mit Ausnahme von Freiburg) sowie in Schwyz und Basel-
Stadt werden die Untersuchungen und Interventionen multiprofessionell, das heisst in Kooperation mit anderen Fachpersonen (mehrheitlich Pflegefachpersonen, aber auch Orthoptistinnen und Orthoptisten), erbracht. In der Westschweiz werden verbreitet «infirmières scolaires» eingesetzt. So beispielsweise im Kanton Waadt, wo vor Schulantritt ein individuelles Gespräch mit dieser spezialisierten Pflegefachperson angeboten wird. Zudem steht diese bei Bedarf für Beratungen und medizinische Leistungen wie Sehtests zur Verfügung. In den acht Kantonen, welche die schulärztlichen Untersuchungen in den privaten Sektor ausgelagert haben, nimmt der schulärztliche Dienst eine Reihe anderer Aufgaben wahr, welche jedoch nicht Gegenstand der vorliegenden Studie waren (z.B. im Kanton Waadt, wo verschiedene Projekte zu Präventionsthemen in den Schulklassen angeboten werden und bei speziellem Bedarf jederzeit ein Termin beim Schularzt oder bei der Schulärztin vereinbart werden kann).

\section{Organisationsmodelle schulärztlicher Untersuchungen in der Schweiz}

Basierend auf den beschriebenen Charakterisierungsdimensionen lassen sich vier Modelle der Organisation schulärztlicher Untersuchungen unterscheiden. Dabei fällt auf, dass der Umgang mit Impfungen innerhalb der Modelle sehr unterschiedlich sein kann.

\section{Klassisches behördliches Modell}

Am häufigsten findet sich das klassische behördliche Modell. Elf Kantone, insbesondere aus der Deutschschweiz, lassen sich diesem Modell zuordnen. In diesen Kantonen beauftragt die kantonale oder kommunale Verwaltung den schulärztlichen Dienst mit der Durchführung der schulärztlichen Untersuchungen. In der Regel werden während der Schulzeit zwei bis drei Untersuchungen bei Kindern und Jugendlichen durchgeführt. Dabei werden insbesondere Gewicht und Grösse gemessen sowie Hör-, Sehvermögen und der Rücken bezüglich Skoliosen geprüft. In der Sekundarstufe wird zudem meist auch ein Beratungsgespräch mit den Jugendlichen geführt.

\section{Reduziertes behördliches Modell}

Drei Kantone sind ähnlich organisiert wie diejenigen im ersten Modell, unterscheiden sich aber durch seltenere und geringere Breite ihrer Untersuchungen. Dieser Typ lässt sich als reduziertes behördliches Modell benennen. 


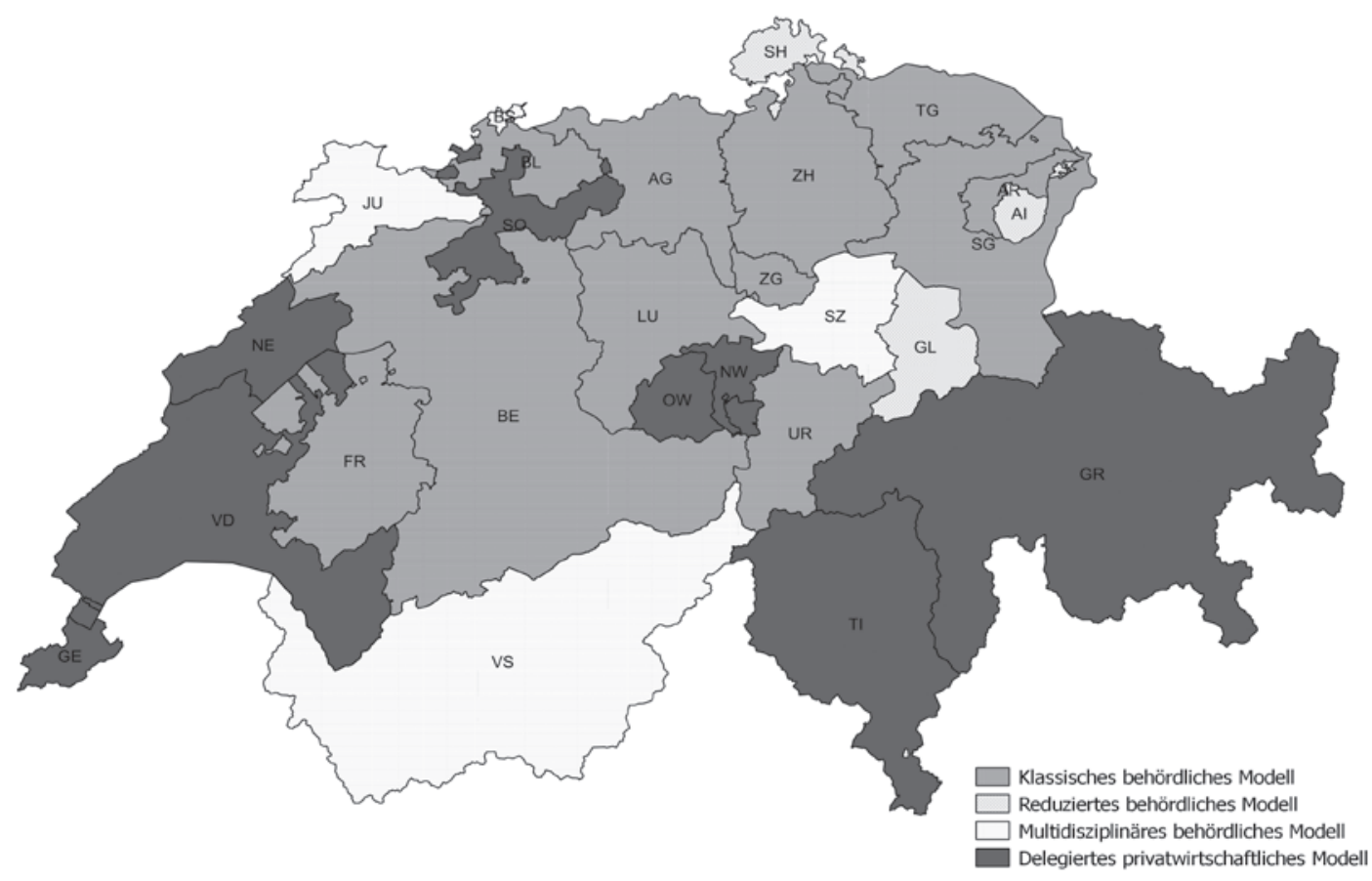

Abbildung 1: Modelle zur Organisation der schulärztlichen Untersuchungen in der Schweiz.

\section{Multiprofessionelles behördliches Modell}

In vier Kantonen wird ein ähnlicher Leistungsumfang wie beim erstgenannten Typ erbracht. Allerdings führt hier ein multiprofessionelles Team von Fachpersonen aus verschiedenen Gesundheitsberufen die Untersuchungen durch (multiprofessionelles behördliches Modell).

\section{Delegiertes privatwirtschaftliches Modell}

Die verbleibenden acht Kantone haben die schulärztlichen Untersuchungen ganz oder zum grossen Teil in den privaten Sektor der Grundversorgung ausgelagert. In der Hälfte dieser Kantone wird eine Bescheinigung über die Durchführung einer vergleichbaren Palette von Abklärungen wie im erstgenannten Typ verlangt. Die Kantone dieses Modells können über einen aktiven schulärztlichen Dienst verfügen, welcher eine Reihe weiterer Leistungen ausser den Untersuchungen erbringt.

\section{Schlussfolgerung}

Wie die vorliegende Studie dokumentiert, sind die schulärztlichen Untersuchungen einschliesslich allfälliger Impfungen in der Schweiz heterogen organisiert und von unterschiedlichem Umfang. Zudem zeigt sich, dass der Bund keine klare Zielsetzung der schulärztlichen Dienste vorgibt und dass auch die öffentlich zugänglichen Informationen auf Kantonsebene diesbe- züglich kaum Klarheit schaffen. Grundsätzlich birgt der schulärztliche Dienst ein grosses Potential, Kinder und Jugendliche flächendeckend in der Schweiz zu erreichen, Versorgungslücken zu schliessen und präventiv wirksam zu sein. Auch für die Berichterstattung zur Kindergesundheit in der Schweiz könnte er einen wichtigen Beitrag liefern [11]. Die Ergebnisse dieser Studie sollen zur Diskussion anregen, wie dieses Potential idealerweise genutzt werden kann. Spannende Fragen sind insbesondere, welche Leistungen erbracht werden sollten, wie eine wirksame und wirtschaftliche Umsetzung zu organisieren wäre und wie sichergestellt werden kann, dass ein relevanter Beitrag zur Versorgung unterschiedlicher - insbesondere vulnerabler - Gruppen geleistet wird $[12,13]$. Die Tatsache, dass sich die Organisation der schulärztlichen Untersuchungen in der Schweiz in vier Modelle gliedern lässt, bietet eine ideale Grundlage für vergleichend angelegte derartige Analysen.

\section{Verdankung}

Wir danken Frau Dr. Tina Huber-Gieske, Mitglied von ScolarMed, und Frau Prof. Dr. med. Julia Dratva der ZHAW sowie den Mitarbeitenden diverser schulärztlicher Dienste, welche die Entstehung dieses Beitrags inhaltlich unterstützt haben.

\section{Disclosure statement}

Es liegt keine Interessenbindung der Autoren/-innen im Zusammenhang mit der Studie vor.

\section{Bildnachweis}

Universität Luzern

\section{Literatur}

Die Literatur findet sich unter www.saez.ch $\rightarrow$ Aktuelle Ausgabe oder $\rightarrow$ Archiv $\rightarrow 2019 \rightarrow 20$. 